\title{
Criminologie
}

\section{Vingt ans de droits des détenus au Québec}

\section{Guy Lemire}

Volume 24, numéro 1, 1991

Les droits des détenu-e-s

URI : https://id.erudit.org/iderudit/017303ar

DOI : https://doi.org/10.7202/017303ar

Aller au sommaire du numéro

Éditeur(s)

Les Presses de l'Université de Montréal

ISSN

0316-0041 (imprimé)

1492-1367 (numérique)

Découvrir la revue

Citer cet article

Lemire, G. (1991). Vingt ans de droits des détenus au Québec. Criminologie, 24(1), 63-76. https://doi.org/10.7202/017303ar

\section{Résumé de l'article}

Discussion of prisoners' rights has often been the centre of attention over the past twenty years or so. While it can help in denouncing the abuse of authority, it is a different matter when it is a question of analyzing the violence between prisoners that is one of the major characteristics of the prison today. It has tended to take refuge in a legalism that leaves no room for more concrete human considerations. By rallying the abolitionists, it has helped to discredit the reeducational prisons that in the sixties were trying to give the prison milieu a more humane image. The outcome is very uncertain.
Ce document est protégé par la loi sur le droit d'auteur. L'utilisation des services d'Érudit (y compris la reproduction) est assujettie à sa politique d'utilisation que vous pouvez consulter en ligne.

https://apropos.erudit.org/fr/usagers/politique-dutilisation/ 


\section{VINGT ANS DE DROITS DES DÉTENUS AU QUÉBEC}

Discussion of prisoners rights has often been the centre of attention over the past twenty years or so. While it can help in denouncing the abuse of authority, it is a different matter when it is a question of analyzing the violence between prisoners that is one of the major characteristics of the prison today. It has tended to take refuge in a legalism that leaves no room for more concrete human considerations. By rallying the abolitionists, it has helped to discredit the reeducational prisons that in the sixties were trying to give the prison milieu a more humane image. The outcome is very uncertain.

Parmi les enjeux carcéraux des deux dernières décennies, le discours des droits des détenus est certes l'un de ceux qui ont le plus attiré l'attention. Cet intérêt se vérifie, non seulement au niveau de la recherche et des écrits, mais aussi sur le plan médiatique. Ce débat ne laisse personne indifférent, d'autant plus que la Charte canadienne des droits et libertés a jeté un nouvel éclairage sur la question. Le temps est donc venu de faire le point, d'analyser quel a été l'apport de cette perspective et quel avenir elle peut avoir.

\section{LE DISCOURS DES DROITS DES DÉTENUS}

Au Québec, ce discours a sans doute atteint son apogée au milieu des années 1970, quand les universitaires et certains groupes sociaux se sont mobilisés contre l'emprisonnement, en ont dénoncé les abus et ont mis de l'avant des propositions destinées «à rendre le milieu carcéral le plus juste et le plus humain» (Landreville, Gagnon et Desrosiers, 1976).

S'il est un dossier qui a marqué cette époque, c'est bien celui de la fermeture du Centre de prévention de Montréal, communément appelé Parthenais. Pendant près de trois ans, un front commun d'une quinzaine d'organismes a travaillé avec acharnement sur ce dossier.

La lutte débuta par de multiples revendications concernant les conditions de détention (...) pour finalement déboucher, après maintes réflexions, constatations et critiques, sur une demande de désaffectation de cette institution. (Gagnon et Dumont, 1976.)

* Professeur, École de criminologie, Université de Montréal, Case postale 6128, Succ. «A», Montréal (Québec) H3C 3J7. 
Le front commun a semblé avoir gain de cause puisqu'il reçut, en 1975, l'avis suivant du ministère de la Justice du Québec:

Les plans et devis pour la construction d'une nouvelle prison devant remplacer Parthenais et Bordeaux débuteront cette année. (Gagnon et Dumont, 1976.)

On ne sait ce qu'il advint du front commun à la suite de ce résultat apparemment satisfaisant mais pour Gagnon et Dumont (1976), il s'agissait «d'une lutte à finir». Les dernières lignes de leur article sont révélatrices:

Il faut souligner en terminant que la lutte est devenue plus large pour l'Office des droits des détenus en ce sens qu'elle ne se restreint pas à une demande de remplacement du centre de prévention, elle implique désormais une évaluation de la détention, eu égard au respect des droits fondamentaux de la personne humaine et eu égard aux significations idéologiques, politiques de l'incarcération dans la société québécoise. Après Parthenais, notre lutte continue (...)

L'avenir a donné raison à ces auteurs sur trois points : 1) à compter de 1976, le discours sur les droits des détenus fut surtout l'affaire de l'Office des droits des détenus (ODD); 2) la lutte fut élargie à l'ensemble des conditions de détention, notamment le tribunal disciplinaire, l'isolement cellulaire et les soins médicaux; 3) la contestation idéologique et politique de l'incarcération est demeurée au premier plan, rejoignant ainsi un discours abolitionniste, important à cette époque.

Les principaux droits ainsi identifiés furent les suivants (Landreville, 1976):

— droits à la vie et à la sécurité de la personne;

- droits à l'égalité devant la loi et à la protection de la loi;

- droits à la liberté de parole et à la liberté de presse;

- droits de ne pas être soumis à des peines ou traitements cruels et inusités;

- droits à une audition impartiale de sa cause (face au processus disciplinaire de l'établissement);

- droits à la présomption d'innocence (pour le prévenu).

Ces droits, on le constate, sont de nature générale et touchent des secteurs clés ou moments névralgiques de l'incarcération. On remarquera également que ces droits sont susceptibles de s'appliquer aux citoyens libres de telle sorte qu'il peut s'avérer difficile, sinon impossible, d'y opposer une fin de nonrecevoir. On ne se surprendra donc pas de constater qu'au cours des quinze 
dernières années, il y a eu bien peu d'opposition au principe de tels droits. Et c'est en s'appuyant sur ces droits que l'ODD a construit ses meilleurs dossiers (suicides, bavures policières); c'est quand il s'en est éloigné (qu'on pense au droit à l'évasion!) qu'il a dérapé et perdu de sa crédibilité.

Quelles conclusions peut-on tirer des vingt ans de la cause? Tout d'abord, que le discours semble s'essouffler. L'ardeur des débuts a cédé la place à une certaine lassitude, Parthenais n'a pas bougé et est plus surpeuplé que jamais, la «lutte à finir» (Gagnon et Dumont, 1976) a cessé, faute de combattants, la mobilisation des organismes sociaux est pratiquement inexistante et on tient à l'université des colloques plutôt inoffensifs sur la surpopulation carcérale. Il faut bien le dire, la cause des droits des détenus, radicale et idéologique, est surtout devenue au Québec l'affaire d'un seul homme, Jean Claude Bernheim.

Ensuite, c'est devenu un discours limité, avec une marge de manœuvre relativement mince. Tant qu'on s'en tient à des énoncés de base susceptibles de s'appliquer à tous les citoyens, dans lesquels ces derniers vont se retrouver ou auxquels ils peuvent s'identifier, le discours passe la rampe, quoique sans susciter d'enthousiasme délirant. Quand on veut aller plus loin et toucher des domaines plus spécifiques à la prison, les apôtres des droits des détenus ont moins de succès. Il existe, à titre d'exemple, un problème sérieux de surpopulation dans les institutions carcérales canadiennes et ce problème dure depuis plusieurs années. Rappelons notamment que la double occupation cellulaire (deux détenus dans une cellule prévue pour une personne) dure depuis au moins cinq ans dans plusieurs pénitenciers fédéraux. Qui s'y intéresse? Rappelons aussi qu'à Parthenais et à Bordeaux, faute de cellules, on loge des détenus dans des corridors ou des dortoirs improvisés. Tout ceci a bien sûr un impact sur la qualité de la vie carcérale: les services ne peuvent être de même qualité, les tensions et frustrations sont vives, les relations sont tendues. Comment faire la démonstration que la qualité de vie a diminué? Cette détérioration n'est pas aussi tangible et mesurable qu'un suicide ou qu'une utilisation exagérée de l'isolement cellulaire. Tout le domaine de l'informel, notamment celui des relations humaines, échappe à la problématique des droits des détenus. À partir du moment où certains droits humains essentiels sont respectés, comment faire la démonstration de l'atmosphère d'une prison, de l'indifférence bureaucratique polie du personnel, de la méfiance entre détenus, en somme de tout ce qui fait qu'un milieu carcéral est vivable ou non? La peine privative de liberté, c'est essentiellement un ensemble complexe de relations humaines entre gardiens et gardés que le discours des droits influence bien peu. Le quotidien de la prison est fait d'autre chose que des abus que tentent de prévenir les droits des détenus. Cette dernière perspective s'occupe finalement de l'exception et non de la règle. Les droits des détenus sont incapables d'apporter une 
solution à un des paradoxes majeurs de l'emprisonnement, à l'aube des années 1990: malgré des améliorations matérielles importantes, malgré un plus grand respect des droits des détenus, il n'a jamais été aussi difficile de «faire du temps» que dans les établissements dorés actuels (Lemire, 1990).

\section{L'ADMINISTRATION ET LES DROITS DES DÉTENUS}

Il y a vingt ans, toute allusion aux droits des détenus inquiétait grandement l'administration pénitentiaire. Dans un milieu aussi réfractaire au changement, un tel discours suscitait des résistances majeures. On n'a qu'à se rappeler combien fut longue la lutte des détenus de l'établissement Archambault pour obtenir une activité aujourd'hui bien établie: les visites-contacts et les visites intimes, dites familiales, d'une durée moyenne de trois jours. Ces activités sont entrées rapidement dans les moeurs carcérales. Non seulement les administrateurs carcéraux ne craignent plus de parler des droits des détenus, mais ils se sont jusqu'à un certain point emparés de ce discours, de telle sorte qu'aujourd'hui les organismes sociaux radicaux n'en ont plus l'exclusivité. If you can't beat them, join them! Le contenu du changement a posé moins de problèmes que son principe.

Voyons ce qu'en dit aujourd'hui le Service correctionnel dans son énoncé de mission (1989):

Cela signifie, en fait, que dans toutes nos actions, nous avons la responsabilité d'agir équitablement envers les délinquants, compte tenu du fait qu'ils conservent les droits des autres membres de la société, sauf ceux qui doivent être limités en raison de la peine qui leur a été imposée. Nous prendrons toutes les mesures pour que l'esprit de la Charte canadienne des droits et libertés soit respecté dans tous nos agissements.

Comparons avec ce que souhaitait Landreville à l'époque (1976):

À mon avis, les détenus doivent conserver tous les droits des autres citoyens, sauf celui de circuler dans la communauté.

Il est difficile de ne pas conclure que ces deux textes se rejoignent sur l'essentiel. Le Service correctionnel du Canada (1989) ajoutera, commentant la valeur fondamentale de sa mission:

Comme nous reconnaissons la règle de droit, nous respectons les droits de tous les individus - délinquants, employé(e)s, et toutes les personnes engagées dans le processus correctionnel.

Elle est donc clairement révolue l'époque où une sentence d'emprisonnement équivalait à une mort civile. La règle de droit et la Charte canadienne 
ont maintenant préséance. Dans son énoncé de mission, le Service correctionnel (1989) affirmera: «Nous prendrons toutes les mesures pour que l'esprit de la Charte canadienne des droits et libertés soit respecté dans tous nos agissements. » Garant et Halley (1990) concluent ainsi leur analyse de l'impact de la Charte: «ll ne fait plus de doute que l'article 7 de la Charte a modifié les garanties juridiques en matière de discipline pénale.»

En ce qui concerne les établissements provinciaux, le règlement relatif aux établissements (1979) établit:

La privation de liberté constituée par l'incarcération et les sanctions décrétées par le comité de discipline sont les seules contraintes pouvant être imposées à la personne incarcérée.

Le règlement spécifie aussi que «le fonctionnaire exerce ses fonctions dans le respect des personnes incarcérées».

Non seulement les administrateurs ont-ils affirmé de tels principes, mais ils ont développé des mécanismes permettant aux détenus d'en appeler des écarts à ces droits. Le service correctionnel possède un système de grief à trois niveaux (établissement, régional, national) dont l'efficacité a été soulignée par le Vérificateur général du Canada (1986). Le détenu peut aussi déborder le Service correctionnel et s'adresser à l'Enquêteur correctionnel. Au niveau provincial, la direction de la détention a prévu dans son règlement (1979) un système de plainte à deux étapes (établissement, direction générale). Le détenu peut aussi aller au-delà des services correctionnels et s'adresser au Protecteur du citoyen.

Certes, on pourrait souhaiter que l'Enquêteur correctionnel rende des comptes au Parlement plutôt qu'au Solliciteur général et que les décisions du Protecteur du citoyen soient exécutoires. Mais, en dernière analyse, cela ne modifierait véritablement pas la situation car les administrations ne font guère d'opposition au principe de ces droits. Tout d'abord, parce qu'ils ont compris que la Charte des droits constituait une réalité incontournable; ensuite, parce qu'ils ont réalisé tout le profit qu'ils pouvaient en tirer, du moins à court terme. Reprenons l'exemple des visites familiales mentionnées auparavant. Après des années d'opposition, le Service correctionnel a évalué rapidement, après quelques mois d'utilisation, tout ce qu'il pouvait obtenir des détenus car, bien sûr, ces nouveaux programmes sont liés à la bonne conduite et ont des effets d'apaisement. Ces nouveaux droits servent en définitive à acheter la paix : aussi aucun directeur d'établissement ne voudrait s'en priver.

Les partisans des droits des détenus ont tellement bien réussi que leur discours a été récupéré et banalisé par l'administration carcérale. 


\section{DROITS ET PRIVILÈGES}

La lutte pour les droits des détenus visait de façon spécifique tout le système de privilèges, si répandu en milieu carcéral, et ce qui en découlait: pouvoir discrétionnaire, rapports de force, abus d'autorité, etc. En s'attaquant à ce système, si brillamment décrit par Goffman (1968), on ne se trompait pas de cible puisqu'on allait au cœur de l'organisation pénitentiaire; en effet, les relations gardiens/gardés prennent véritablement vie autour de ces négociations, tolérances, soumissions, récompenses et punitions (Lemire, 1990). Ce faisant, on laissait de côté le fait que le système de privilèges constituait en soi une limite au pouvoir des gardiens: si ceux-ci avaient été en situation de pouvoir absolu ou total (pour reprendre le terme de Goffman), ils n'auraient pas été dans l'obligation de faire les concessions que l'on sait pour obtenir la collaboration des détenus. Le système de privilèges établit des rapports de réciprocité et de dépendance qui, s'ils ne font pas disparaitre les inégalités, établissent que les gardiens ont jusqu'à un certain point besoin des détenus pour assurer le bon fonctionnement des établissements carcéraux (Lemire, 1990). On ne sait pas si les partisans des droits des détenus visaient à rendre ces rapports égalitaires (ou les plus égaux possible) ou s'ils voulaient plus simplement s'assurer d'en faire disparaitre le caractère arbitraire et incertain, de façon à ce que les règles du jeu soient clairement établies.

Il en résulte, quelque vingt ans plus tard, un système de privilèges transformé et des relations modifiées. Un nouveau concept, celui de detotalization (Stasny et Tyrnauer, 1982), a fait son apparition: il désigne l'éclatement de l'établissement total, la fin de l'ordre traditionnel de la prison. À travers l'analyse de la cantine (magasin de l'établissement où le détenu peut se procurer des biens de consommation), Seyler (1985) a mis en évidence qu'à la frugalité légendaire des cantines ont succédé des centaines d'articles parmi lesquels figurent en bonne place des objets de luxe. La cantine témoigne à sa façon qu'il faut désormais davantage de privilèges pour obtenir la soumission des détenus. Les privilèges semblent engagés dans une spirale inflationniste où plus on en a, plus on en veut (Lemire, 1990). Seyler (1985) écrit que «l'extension des cantines (...) c'est l'obligation de gérer des relations devenues plus complexes».

La conséquence de cet essor magistral des privilèges, c'est «l'affaissement de la clôture» (Seyler, 1985). Le développernent des privilèges diminue la spécificité de la prison et rapproche graduellement cette dernière de la société. Le détenu peut maintenant avoir accès aux produits d'une société de consommation: il a, de sa cellule, les mêmes informations et les mêmes divertisse- 
ments, grâce au téléviseur et au système de son, que le citoyen dans le salon de sa maison; il peut lire les mêmes journaux et revues et suivre les mêmes modes de coiffure et d'habillement; il peut même occasionnellement retrouver la sexualité de son choix. Le niveau de vie de certains détenus dépasse celui de plusieurs citoyens démunis.

Auparavant, il était souvent de mise de comparer la prison à une ville autonome; le milieu carcéral traditionnel se suffisait à lui-même et n'avait pas besoin de la société pour fonctionner. Aujourd'hui, on constate qu'une brèche a été ouverte, qu'une osmose s'est établie et que la société est entrée en prison. Le milieu carcéral se voit privé d'une partie de son caractère d'exception et semble irrésistiblement poussé vers la normalité (Lemire, 1990). D'où la conclusion essentielle de Seyler (1985): «L'institution n'est plus la source de tout ce qui fait sens dans la vie carcérale et son caractère total est entamé.»

L'essor des privilèges fait en sorte qu'ils se rapprochent de plus en plus des droits, qu'ils se négocient à des niveaux plus élevés de la hiérarchie organisationnelle et que le gardien, par conséquent, se trouve graduellement dépossédé de son principal outil de travail. Le détenu ayant de moins en moins besoin du gardien pour "vivre en prison», la relation gardiens/gardés perd de sa signification, elle se vide progressivement de son contenu et on ne sait pas encore ce qui lui succédera. Les gardiens, plus que jamais en situation d'incertitude, optent pour une stratégie de retrait: on les retrouve de plus en plus dans les postes de contrôle et les bureaux, laissant les détenus se débrouiller entre eux (Conrad, 1977). Ce même Conrad (1977) a magnifiquement illustré cette évolution en démontrant que, d'une situation de couple (gardien-gardé), la prison est passée à un triangle (gardien-gardé-gardé), dans lequel l'interlocuteur principal du détenu n'est plus le gardien mais l'autre détenu.

La direction se trouve également affaiblie (Jacobs, 1976; Conrad, 1977). L'entrée de la société en prison a fait en sorte qu'un ensemble de groupes aux intérêts différents, sinon contradictoires, a fait son apparition et tente d'influencer la gestion carcérale (Ohlin, 1960). Tout se passe comme si plusieurs groupes et individus tiraient une même couverture dans des directions tout à fait différentes: le résultat dépend davantage du jeu des forces en présence que de la concertation et de la rationalité (Lemire, 1990).

À partir du moment où les administrations locales et centrales ont grugé une partie de son pouvoir décisionnel, où les élus du peuple ont imposé leur présence et leurs orientations, où les tribunaux ont contrôlé sa gestion, où les médias ont mis l'accent sur ses erreurs et ses échecs, la direction a perdu une grande partie de l'immense pouvoir qu'elle détenait (Stasny et Tyrnauer, 1982). 
Pendant ce temps, à l'intérieur, la situation s'est compliquée: groupes de détenus aux intérêts plus diversifiés, syndicalisation du personnel, fractionnement du personnel. La direction se retrouve donc face à une gestion interne plus complexe avec des pouvoirs diminués. Cette situation fait d'elle un médiateur plutôt qu’un décideur (Lemire, 1990).

Puisque les conditions matérielles de détention se sont améliorées, puisque les privilèges ont grandement augmenté, puisque les interlocuteurs des détenus (gardiens et direction) sont devenus plus vulnérables et moins susceptibles d'abus de pouvoir, on pourrait s'attendre à ce que l'univers des détenus soit devenu plus vivable et humain.

Au contraire, ce monde se présente comme conflictuel et désorganisé, et rien ne l'illustre mieux que l'analyse de la violence interpersonnelle. On se rappellera que, traditionnellement, il s'est agi de la violence des gardiens sur les détenus; or celle-ci a considérablement diminué à partir de l'aprèsguerre pour devenir maintenant un phénomène très limité (Lemire, 1990). La violence des détenus sur la personne des gardiens a fait son apparition, mais demeure modeste (Bowker, 1980). À l'heure actuelle, la véritable violence carcérale est celle qui se déroule entre détenus (Conrad, 1976; Lockwood, 1980; Conrad, 1977). Les homicides de détenus par d'autres détenus ont atteint, au cours des deux dernières décennies, tant aux États-Unis (Sylvester et al., 1977) qu'au Canada (Jayewardene et Doherty, 1985), des sommets inégalés.

On peut trouver un indice sûr de cette violence dans l'essor des clientèles de protection, c'est-à-dire des détenus qui à leur propre demande sont placés à l'écart de la population carcérale. Traditionnellement, la clientèle de protection pouvait toucher de 1 à 2 pour cent des détenus. Or, depuis quinze ans, les choses changent et le nombre de détenus demandant protection s'accroît de façon importante (Conrad, 1977). Dans l'État de l'llinois, 17 pour cent de la population carcérale a choisi la protection (Anderson, 1980). Au Canada, on construit de nouveaux établissements pour cette seule clientèle.

En outre, les motifs semblent être plus flous: aux motifs traditionnels (délations, dettes, délits sexuels) s'est ajoutée l'incapacité de s'entendre avec les autres détenus (Anderson, 1980). Il y a une crainte diffuse et une peur des autres, insupportables, qui font considérer l'étiquette de protection comme un moindre mal (Conrad, 1977; Lockwood, 1985). Les relations interpersonnelles sont devenues tendues, imprévisibles; même les leaders sont touchés (Conrad, 1977).

En somme, un nombre élevé de détenus préferent dorénavant la protection des gardiens au contact des autres détenus. Comment se fait-il que les ennemis 
d'hier deviennent les protecteurs d'aujourd'hui? Que se passe-t-il pour que les relations avec les gardiens soient considérées, par un nombre croissant de détenus, que rien ne destinait à ce cheminement, comme un moindre mal? Car c'est souvent dans les établissements à sécurité élevée que se recrute la clientèle de protection, donc parmi les détenus théoriquement les plus irréductibles (Lemire, 1990).

Le moins que l'on puisse dire, c'est que l'importance grandissante de la protection témoigne d'un malaise certain dans l'univers des détenus, et on ne peut éviter d'établir des liens entre cet univers de peur et l'importance de la violence carcérale que nous venons de souligner.

Nous nous retrouvons de nouveau face à cet immense paradoxe: les conditions objectives de détention n'ont jamais été meilleures, nous n'avons jamais été aussi près de la reconnaissance des droits des détenus, mais le monde des détenus demeure singulièrement désorganisé et violent. De fait, il l'est peut-être plus que jamais. L'affaissement de l'établissement totalitaire et l'avènement des droits de détenus n'auront-ils eu pour effet, en dernière analyse, que de remplacer un tyran par un autre? La tyrannie de la minorité est-elle plus légitime que celle de la majorité? Il sera intéressant de voir si les organismes spécialisés dans les droits de la personne et des détenus dénonceront les abus des détenus sur d'autres détenus avec la même vigueur qu'ils ont dénoncé ceux des gardiens.

Il en va de la crédibilité de ces organismes, comme on l'a vu lors de la crise autochtone de l'été 1990. Quand le préjugé favorable à la minorité empêche d'analyser l'ensemble de la réalité de façon objective et rigoureuse, on ne doit pas se surprendre de ne plus être écouté.

Les gens du crime organisé et les membres des gangs de motards sont certes, à l'intérieur de l'univers délinquant, ceux qui bafouent le plus les droits de la personne. Les bavures policières et carcérales ne font pas le poids et, même si on les éliminait, cela ne diminuerait que de façon marginale la violence du monde délinquant. Et pourtant, quel organisme s'est levé pour dénoncer ces abus du milieu interlope? Tout se passe comme si les lésions des droits essentiels ne méritaient d'être dénoncées que lorsqu'elles sont commises par l'autorité légitime. Il faudrait alors spécifier qu'on s'intéresse moins au respect des droits qu'aux abus des détenteurs du pouvoir formel.

Dans la meilleure des hypothèses, le discours des droits des détenus aura contribué, avec celui d'autres groupes d'intérêt bien sûr, à l'effritement de l'institution totalitaire et à l'affaiblissement de l'autorité formelle. Mais son incapacité à intervenir dans l'univers des détenus nous laisse entrevoir qu'il n'est guère apte à résoudre «le problème de ce que peut et doit être une société 
libérée de la menace totalitaire» (Ferry et Renaut, 1985). Si le discours des droits s'est essoufflé, c'est qu'avec la fin du totalitarisme, il ne semble pas en mesure d' «affronter la question d'une société juste, égale et libre» (Ferry et Renaut, 1985). Sa lutte CONTRE ne s'est pas transformée en lutte POUR.

\section{LIMITES DU JURIDISME}

L'une des caractéristiques majeures de notre époque, c'est l'explosion des libertés et l'éclatement des mécanismes de contrôle traditionnels (Crozier et Friedberg, 1977). Il est bien sûr que le discours des droits de la personne y a contribué, en prison comme en société. En formalisant les règles du jeu et en circonscrivant les rapports de pouvoir, on a mis à la disposition des citoyens les moins privilégiés des ressources essentielles assurant la satisfaction des besoins fondamentaux. Mais, comme dit Dufresne (1987): «(La règle de droit) fait passer le processus avant le résultat, la compétence avant la conscience, la forme avant la substance.» Dufresne (1987) cite Henri Brun, professeur à la faculté de droit de l'université Laval:

Une Charte omniprésente, parce qu'interprétée dans l'abstrait et l'absolu, entraînera l'usure des droits qu'elle est censée servir. Plus encore, elle sera source d'une injustice croissante au profit des plus forts, des plus puissants, des mieux organisés et des mieux nantis, au lieu d'assurer d'abord et avant tout, comme elle se doit, la protection des plus vulnérables. Bien loin d'engendrer une société plus juste, plus sécuritaire et plus douce, c'est une société d'affrontements et de conflits qu'elle favorisera, une société fondée sur la réclamation maximale des droits et l'accomplissement minimal des devoirs.

Le légalisme ne peut régler la qualité des rapports humains puisqu'il ne s'intéresse pas au contenu. La personne n'existe pour lui que comme entité juridique, abstraite et désincarnée. Pour reprendre Dufresne (1987), la règle de droit est incapable de dépasser une «rationalité technique». À la limite, il n'y a rien de moins humain que le juridisme. Voilà qui place en meilleure perspective ce que nous affirmions plus tôt dans cet article: même si les droits des détenus n'ont jamais été aussi respectés que maintenant, même si les conditions matérielles de détention n'ont jamais été meilleures, il est plus difficile que jamais de purger une peine privative de liberté.

Les administrations pénitentiaires ne se privent pas de cette «rationalité technique», si facile pour elles à contrôler. Elles se débrouillent beaucoup plus aisément dans cette mécanique que dans la gestion de rapports humains de qualité. Il est plus simple de respecter certains droits que de rendre les prisons «vivables». On peut même se demander si le discours des droits, si brillam 
ment récupéré par l'administration carcérale, n'est pas devenu autre chose que de la poudre aux yeux servant à détourner l'attention des vrais problèmes.

Dès lors, une question mérite d'être posée: le discours des droits a-t-il bien eu, en prison, l'utilité que nous lui avons prêtée jusqu'à maintenant? $\mathrm{Ce}$ n'est pas évident. Pour mieux comprendre la situation, il faut se reporter à 1970. Le milieu pénitentiaire venait de connaître deux décennies de développement intense. C'est en effet durant la période de l'après-guerre que se sont développés les établissements à sécurité réduite, lesquels constituaient en soi une amélioration notable des conditions de détention si on les compare aux forteresses à sécurité maximale de l'époque. Ces nouveaux développements s'appuyaient sur une volonté clairement affirmée de mettre l'accent sur la réhabilitation et la réinsertion sociale des délinquants. Si on utilise la typologie de Stasny et Tyrnauer (1982), il s'agissait du passage de la prison de l'entreposage à l'établissement rééducatif.

Et c'est la prison rééducative qui, la première, a mis fin à la mort civile du détenu en le définissant comme un citoyen susceptible de réintégrer la société avec succès. C'est elle qui a modifié le rapport traditionnel gardiens/ gardés en introduisant le professionnel des sciences humaines destiné à aider le détenu. C'est de cette époque aussi que datent la fin de l'autosuffisance totalitaire du milieu carcéral et l'entrée de la société en prison en tant qu'élément essentiel de la réinsertion sociale.

On ne se trompe guère en affirmant que la période de la prison rééducative est responsable de ce qui constitue encore aujourd'hui les principales améliorations qu'a vécues ce milieu. Si la prison a pu avoir une certaine humanité, c'est à cette époque. Les droits des détenus n'ont donc rien inventé: ils ont plutôt profité de conditions favorables créées par d'autres. Et, en franchissant une porte déjà ouverte, ils ont pu développer certains dossiers intéressants, mentionnés dans la première partie de l'article. Leur erreur fut sans doute de joindre la perspective abolitionniste. C'était bien tentant puisqu'il s'agissait d'un discours dominant durant les années 1970 . Or, ce discours abolitionniste avait parmi ses cibles principales la prison rééducative et il est en partie responsable de la défaveur qui est le lot de la réhabilitation et de la réinsertion sociale depuis lors. En privilégiant une perspective abolitionniste et, par conséquent, un discours anti-réhabilitation, il a contribué à neutraliser les sciences humaines et à renforcer la perspective légale. Il a fait en sorte que l'avocat devienne plus important que le criminologue. Au pouvoir discrétionnaire du dernier, il a préféré la rationalité abstraite du premier. Dès lors, faut-il se surprendre que l'humanité des prisons soit si peu évidente à l'heure actuelle? Même les criminologues jouent maintenant le jeu si rassurant du légalisme et de la bureaucratie. 
À la prison rééducative a succédé l'anarchie actuelle dont la violence entre détenus est une des principales manifestations. Le discours des droits des détenus, par son choix de l'abolitionnisme, a contribué à défaire d'une main ce qu'il tentait de construire de l'autre. Il a en quelque sorte sapé sa propre action.

Au-delà des niveaux matériel et technique, qui peut parler de progrès au cours des vingt dernières années? Sur le plan humain, il convient davantage de parler de régression. En s'éloignant de la prison rééducative, on a mis de côté le seul «souffle humaniste» (Pires, 1987) à avoir inspiré la gestion de la prison. Aux détenus-numéros des établissements totalitaires, on a substitué des abstractions juridiques. Les tenants des droits des détenus se sont davantage préoccupés de l'être humain que des êtres humains. Des perspectives légales, qui ne sont somme toute que des moyens, sont devenues des fins. De quel progrès s'agit-il?

\section{UN DISCOURS AMBIGU ET UN RÉSULTAT INCERTAIN}

Avec le recul, on comprend que le discours des droits des détenus ne s'intéressait que partiellement aux détenus. C'est que sa véritable cible était de pius en plus la prison. On se préoccupait plus d'un système que des personnes. "C'est l'existence même de la prison qu'il faut maintenant remettre en question» (Landreville, Gagnon et Desrosiers, 1976). En effet, se contenter de dénoncer les erreurs et les abus permet à la prison de les corriger et finalement de s'améliorer: une telle optique, réformiste, ne fait que renforcer l'existence de la prison. Ce n'est pas ce qui intéresse les abolitionnistes, au contraire. Pour justifier l'abolition de la prison, on doit démontrer son inutilité et ses atrocités. Dans cette perspective, il n'est pas souhaitable que la prison s'améliore : cela contredit l'argumentation. C'est sans doute pour cette raison qu'on n'a guère entendu les tenants des droits des détenus vanter les mérites de certains programmes d'étude ou socio-culturels, dont même les détenus reconnaissent la qualité (Pinsonneault, 1985). Il serait sans doute dans l'intérêt des détenus que l'on mette l'accent sur les réussites du système carcéral et qu'on fasse pression pour que ces succès soient développés. L'intérêt des abolitionnistes ne va pas dans ce sens. On privilégie un objectif à plus long terme, même si, dans l'immédiat, on doit négliger des gens. Pour les tenants de l'abolitionnisme et les radicaux des droits des détenus, les vivants offrent moins d'intérêt que les morts à partir desquels ils construisent des dossiers-chocs (suicides, homicides par les policiers). Les deux discours, droits des détenus et abolitionnisme, ne sont donc pas obligatoirement compatibles. À court terme, ils sont même souvent contradictoires. 
À long terme, rien n'est joué. Il se peut que l'accroissement des privilèges et des droits exerce une pression telle sur la prison qu'on assiste à son éclatement. Les abolitionnistes auraient alors eu raison. Il se peut aussi que l'éclatement totalitaire mène à un autre modele de gestion de la peine privative de liberté. Certains établissements rééducatifs pour jeunes délinquants en font la démonstration. On ne peut parler, par exemple, pour Boscoville d'établissement coencitif totalitaire, et pourtant c'est essentiellement d'une prison qu'il s'agit. Certes, on ne peut prétendre que les prisons de l'avenir pourront toutes être de ce calibre. Mais ce que cet exemple permet d'établir, c'est que la fin du totalitarisme en milieu carcéral ne mène pas obligatoirement à la fin de l'emprisonnement.

En conclusion, si on ne peut nier que le discours des droits des détenus ait produit certains résultats positifs, son utilité a finalement été plutôt réduite et, sous certains aspects, il y a lieu de parler d'effets contre-productifs. Le discours ne semble pas en mesure de jouer un rôle déterminant dans la vie quotidienne de la prison et encore moins de combler le vide actuel qu'on y observe et qui n'est guère à l'avantage des détenus.

Cela annonce peut-être la fin d'une époque (et nécessitera d'ailleurs une analyse séparée). Vingt ans de discours abolitionniste, d' «alternatives» à l'emprisonnement et de droits des détenus (puisque finalement tout cela se tient) ont mené à des prisons plus surpeuplées et toujours aussi conflictuelles, sans véritable vocation, où domine le plus souvent l'entreposage humain. Le temps est venu de demander des comptes. Nothing works! On a été moins patient avec la rééducation.

\section{BIBLIOGRAPHIE}

ANDERSON, D. C. (1980), "The Price of Safety», Corrections Magazine, vol. 6, $\mathrm{n}^{\circ} 4$, pp. 6-15.

BERNHEIM, J. C. (1987), Les Suicides en prison, Montréal, Éditions du Méridien.

BOWKER, L. H. (1980), Prison Victimization, New York, Elsevier.

CONRAD, J. P. (1976), «The Beast Behind the Wall», dans A. K. Cohen, G. F. Cole, R. G. Bailey (éd.), Prison Violence, Toronto, Lexington Books.

CONRAD, J. P. (1977), «The Survival of the Yearful», dans J. P. Conrad et S. Dinitz (éd.), In Fear of Each Other, Toronto, Lexington Books.

CROZIER, M., FRIEDBERG, E. (1977), L'Acteur et le système, Paris, Éditions du Seuil.

DIRECTION GÉNÉRALE DE LA PROBATION ET DES ÉTABLISSEMENTS DE DÉTENTION (1979), Lois et règlements relatifs aux personnes incarcérées, Québec, Éditeur officiel du Québec. 
DUFRESNE, J. (1987), Le Procès du droit, Montréal, Institut québécois de recherche sur la culture.

FERRY, L., RENAUT, A., (1985), Des droits de l'homme d̀ l'idée républicaine, Paris, Presses universitaires de France.

GAGNON, A., DUMONT. H. (1976), «Parthenais, début d'une lutte», Criminologie, vol. IX, $\mathrm{n}^{\mathrm{os}} 1-2$, pp. 163-188.

GARANT, P., HALLEY, P. (1990), "L'article 7 de la Charte canadienne et la discipline carcérale», Revue générale de droit, vol. 20, juin 1990, pp. 599-646.

GOFFMAN, E. (1968), Asiles, Paris, Minuit.

JACOBS, J. B. (1976), «Prison Violence and Formal Organization», dans A. K. Cohen, G. F Cole et R. G. Bailey (éd.), Prison Violence, Toronto, Lexington Books.

JAYEWARDENE, C. H. S., DOHERTY, P. (1985), «Individual Violence in Canadian Penitentiaries", Revue canadienne de criminologie, vol. 27, $\mathrm{n}^{\circ}$ 4, pp. 429-439.

LANDREVILle, P., GAGNON, A., DESROSIERS, S. (1976), Les Prisons de par ici, Montréal, Éditions Parti Pris.

LANDREVILLE, P. (1976), «Les détenus et les droits de l'homme», Criminologie, vol. IX, $\mathrm{n}^{\mathrm{os}}$ 1-2, pp. 107-117.

LEMIRE, G. (1990), Anatomie de la prison, Montréal, Presses de l'Université de Montreal.

LOCKWOOD, D. (1980), Prison Sexual Violence, New York, Elsevier.

LOCKWOOD, D. (1985), «Issues in Prison Sexual Violence», dars M. Braswell, S. Dillingham et R. Montgomery ( $£ d$.), Prison Violence in America, Cincinnati, Anderson.

OHLIN, L. (1960), «Conflicting Interests in Correctional Objectives», dans R. Cloward, et al. (éd.), Theoretical Studies in the Social Organization of the Prison, New York, Social Science Research Council.

PINSONNEAULT, P. (1985), "L'abandon de la carrière criminelle», Criminologie, vol. XVIII, $\mathrm{n}^{\circ} 1$, pp. 85-116.

PIRES, A. (1987), «La réforme pénale au Canada: l'apport de la Commission canadienne sur la détermination de la peine», Criminologie, vol. $\mathrm{XX}, \mathrm{n}^{\circ} 2$, pp. 11-55.

SERVICE CORRECTIONNEL DU CANADA (1989), La Mission du Service correctionnel du Canada, Ottawa, Ministère des Services et Approvisionnements.

SEYLER, M. (1985), La Consommation dans les établissements pénitentiaires, Paris, CESDIP

STASTNY, C., TYRNAUER, G. (1982), Who Rules the Joint?, Toronto, Lexington Books.

SYLVESTER, S. F., REED, J. H., NELSON, D. O. (1977), Prison Homicide, New York, Spectrum.

VÉRIFICATEUR GÉNÉRAL DU CANADA (1986), Rapport du Vérificateur général du Canada à la Chambre des communes, Ottawa, Ministère des Approvisionnements et Services. 